Ann. Zootechn., I979, 28 (2), I85-I00.

\title{
Teneur en androsténone des graisses de jeunes porcs mâles entiers issus de croisement de type "Camborough "
}

\author{
M. BONNEAC, B. DESMOULIN \\ avec la collaboration technique de P. Peniste, P. Grandsart et R. Chatifik \\ Station de Recherches sur l'Elevage des Porcs \\ Centre national de Recherches Zootechniques, I.N.R.A., \\ 78350 Jouy-en-Josas (France)
}

\begin{abstract}
Résumé
La teneur en androsténone (5 $\times$ androst-16-ene-3-one) du tissu gras dorsal a été mesurée chez 77 pores mâles entiers de type "Camborough " abattus à roo kg de poids vif aux environs de I 50 jours d'âge. Les teneurs en stéroïde inférieures à I ppm, comprises entre I et 2 ppm on supérieures à 2 ppm concernent respectivement 58,30 et $\mathrm{I} 2 \mathrm{p}$. I oo des animaux. Aucune relation significative n'est mise en évidence entre le niveau de stockage d'androsténone dans les graisses d'une part et le poids vif, le poids des testicules ou la composition corporelle des animaux d'autre part. Compte tenu des liaisons entre la teneur en stéroïde et les qualités olfactives des graisses et des viandes, une proportion importante des viandes présente des risques élevés de défauts d'odeur lors de la consommation en frais, en dépit du jeune âge des animaux.
\end{abstract}

\section{Introduction}

En race pure, les défauts d'odeur sexuelle des viandes de jeunes porcs mâles entiers sont peu fréquents chez les types conventionnels Large White ou Landrace. A roo $\mathrm{kg}$ de poids vif, vers I 80 jours d'âge, ils concernent I 5 à $20 \mathrm{p}$. Ioo des effectifs (Desmoulin et Rhodes, I975). Par contre, dans le cas des porcs de type hypermusclé et en particulier de race Piétrain, les défauts d'odeur sexuelle prononcée peuvent toucher la majorité des jeunes mâles (BonNeAu, DEsmoulin et Dumont, I979). Corrélativement, les niveaux de stockage dans les graisses du principal composé responsable des odeurs sexuelles $(5 \alpha$ androst-I6-ene-3-one ou androsténone) sont très élevés chez ces animaux. Ile ce point de vue, les populations de porcs mâles entiers issus de croisement ont été à ce jour peu étudiées.

Chez le jeune porc mâle, la teneur en androsténone des graisses augmente avec 1'âge, en particulier à partir de Ioo à I40 jours (CLAUS I975, ANDRESEN I976). 
L'abattage des porcs s'effectuant le plus souvent à poids constant, on peut se demander quels seraient les niveaux de stockage d'androsténone lorsque des animaux à croissance très rapide sont abattus très jeunes aux stades habituels d'abattage.

Le présent essai concerne un échantillon de jeunes porcs mâles entiers de type "Camborough ", résultant du croisement de lignées pures Large White ou Landrace, abattus aux environs de roo $\mathrm{kg}$ de poids vif et de $5_{50}$ jours d'âge. L'étude de la composition des carcasses et de la fréquence des défauts d'odeur sexuelle a fait l'objet d'un précédent article (Bonneau, IEsmoulin et PawLak, I977). Cette présentation rapporte les variation du stockage de l'androsténone dans les graisses et l'étude des relations entre la teneur de ce stéroïde et les critères de composition corporelle ou de qualité organoleptique des viandes.

\section{Matériels et méthodes}

Les 77 animaux étudiés étaient élevés en semi plein air par groupes de 30, à partir de $30 \mathrm{~kg}$ de poids vif. Ils ont été abattus à un poids moyen de $100,6 \mathrm{~kg}$, entre I4I et I 66 jours d'âge au cours de 7 répétitions successives entre mai I 976 et mai r977. Les contrôles suivants ont été effectués :

- pesée des testicules;

- estimation de la composition corporelle (épaisseurs de lard, découpe parisienne, surface du muscle long dorsal);

- mesures de qualité des viandes ( $\mathrm{pH}$ des muscles adducteur et long dorsal $24 \mathrm{~h}$ post mortem);

- notation par un jury de laboratoire de l'intensité des odeurs sexuelles au cours du chauffage des graisses dorsales selon un procédé déjà décrit (BONNEAU, TAssencourt, Desmoulin, I975);

- enquête d'acceptabilité auprès d'un jury de consommateurs des côtelettes des I7 pores de la dernière répétition;

- détermination de la teneur en androsténone du tissu adipeux dorsal à 1'aide de la méthode radioimmunologique mise au point par CrAUs (I974).

\section{Résultats et discussion}

\section{I. - Teneur en androsténone des graisses}

La teneur en androsténone du tissu adipeux dorsal s'élève en moyenne à I,I $6 \mathrm{ppm}$. En outre, comme le montre la figure $I$, les variations individuelles sont très importantes $(\mathrm{C} . \mathrm{V} .=72 \mathrm{p}$. IOO). Des teneurs inférieures à $\mathrm{x}$ ppm sont relevées chez 58 p. Ioo des animaux alors que 30 et I 2 p. Ioo de l'effectif sont situés respectivement entre I et $2 \mathrm{ppm}$ et au-delà de $2 \mathrm{ppm}$. Une telle répartition des animaux, de type lognormale $\left(\mathrm{X}_{2}^{2}=\mathrm{I}, 04 ; \mathrm{P}>0,50\right)$ est semblable à celles observées par ClaUS (I977) ou par MaIMFORS et LUNDSTROM (Communication personnelle) chez des animaux plus âgés abattus à des poids vifs équivalents. Compte tenu du 


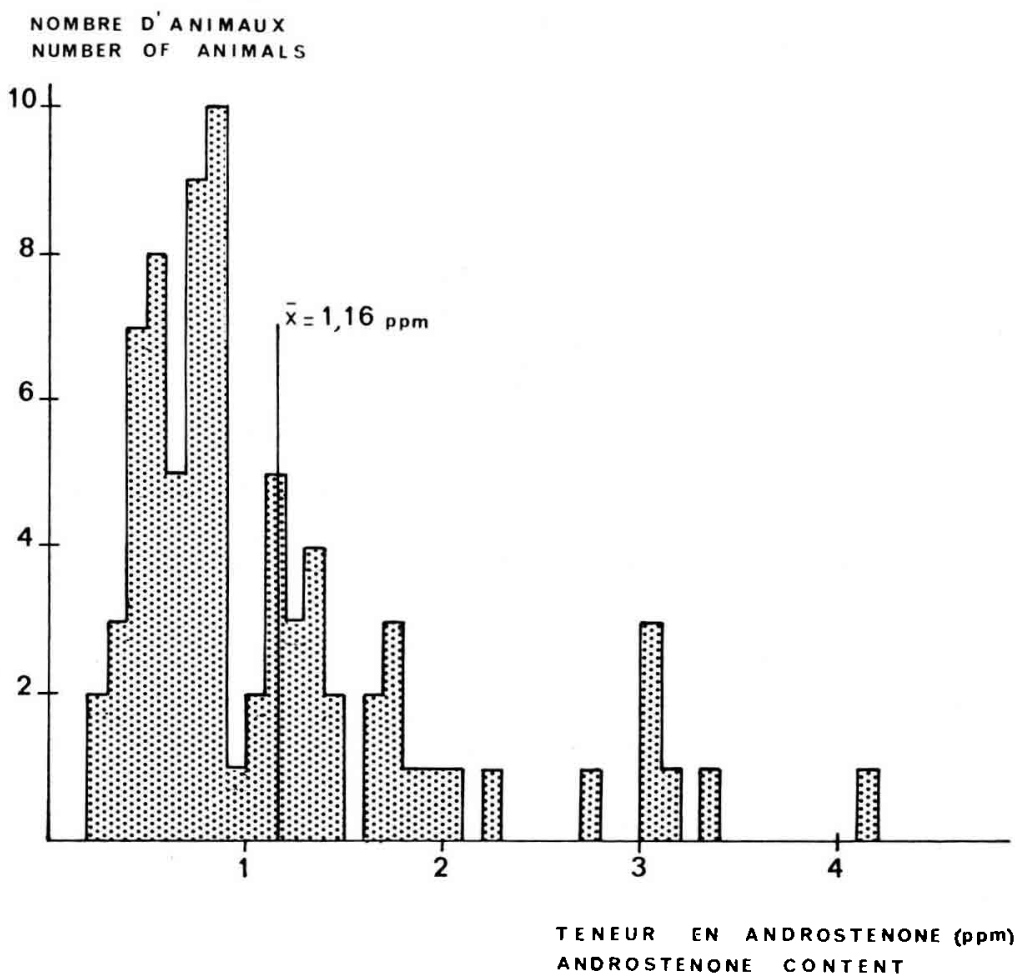

FIG. I. - Répartition des porcs mâles entiers sclon la teneur en androsténone de tissu adipeux. Distribution of chtive males according to backlat androstonone content.

jeune âge des animaux, le niveau moyen de stockage d'androsténone apparaît donc élevé. Ce résultat rejoint les conclusions de WALKER, RoBB et PATTERson (I977) qui ont noté, dans le cas d'animaux abattus à $85 \mathrm{~kg}$ de poids vif, des défauts d'odeur plus fréquents chez des porcs jeunes (I5o jours) que chez leurs contemporains plus âgés (I 86 jours) ayant présenté une croissance plus lente.

\section{II. - Relations anec le poids vif et la composition corporelle}

La teneur en androsténone des graisses n'est pas reliée au poids vif des animaux $(r=+0,02 ; \mathrm{NS})$. Ce résultat semble à première vue en contradiction avec l'augmentation de la teneur en androsténone au cours de la vie du jeune animal (Claus, I975). Mais Axdresen (I 976) a pu montrer que l'âge à partir duquel une augmentation rapide de la teneur en ce stéroüde est observée varie entre roo et I 80 jours d'âge pour des animaux de même race élevés dans les mêmes conditions. Ceci expliquerait donc, dans les limites étroites des stades d'abattages considérés, la variation individuelle très importante des niveaux de stockage d'androsténone, sans relation avec le poids vif des animaux.

Compte tenu de l'effet anabolisant des hormones sexuelles d'une part, et des relations étroites entre ces hormones et l'androsténone d'autre part (GROTH et CLAUS, I977; CIAUS, I977), on pourrait s'attendre à une liaison entre les performances des animaux et les niveaux de stockage d'androsténone. Cependant, les 
coefficients de corrélation entre la teneur des graisses en ce stéroïde et les critères de composition corporelle (rapport : longe /bardière : $r=-0, \mathrm{I} 8$; surface du muscle long dorsal : $r=-0, \mathbf{1 6}$; épaisseur de lard au rein : $r=-0$, Io) ou de qualité de viande ( $\mathrm{pH}$ des muscles adducteur : $r=-0,25$ et long dorsal : $r=+0,0 \mathrm{I}$ ) sont faibles et non significatifs. De même ANDRESEN et BAKKE (I975) et ANDRESEN (I976) n'observent aucune différence significative entre les teneurs en androsténone de 3 lignées de porcs maigres à croissance rapide, gras à croissance lente ou témoins non sélectionnés.

Cette indépendance entre la teneur en androsténone des graisses et les critères de production peut faire l'objet de plusieurs hypothèses :

- la teneur en androsténone des graisses, résultant de l'équilibre entre la production et l'élimination de ce stéroïde ne reflèterait pas l'intensité de la synthèse des stérö̈des testiculaires. La relation mise en évidence par GRoTH et $C_{\text {LAUS }}$ (I977) entre la teneur en androsténone des graisses et la concentration en testostérone du plasma de la veine spermatique est certes assez étroite $(r=0,7 \mathrm{I}$; $\mathrm{P}<0,001)$ mais elle laisse inexpliquée $50 \mathrm{p}$. roo de la variabilité totale du niveau de stockage d'androsténone;

- 1'effet anabolisant des androgènes sur le muscle squelettique est démontré (GRIBSBY, BERGEN et MERKEL, I976) mais il est moins marqué que dans le cas du muscle levator ani (BUREsova et GuTMAN, I97I) généralement utilisé pour mettre en évidence l'action myotrophique des hormones sexuelles (EisENBERG et GORDON, I950).

\section{III. - Relations avec le poids des testicules}

Le poids des 2 testicules s'élève en moyenne à $454 \mathrm{~g}$. La variabilité individuelle assez élevée (C.V. = I9,7 p. I00) n'est que partiellement expliquée par les variations du poids vif $(r=+0,52 ; \mathrm{P}<0, \mathrm{or})$.

Le coefficient de corrélation entre la teneur en androsténone des graisses et le poids des testicules n'atteint pas la limite de signification $(r=+0$,I $(9)$. Mais on sait que le testicule de verrat ne contient que $I_{5}$ à $20 \mathrm{p}$. Ioo de tissu interstitiel (Dors'T et SAJonski, I 974), seul responsable des sécrétions endocrines. Grotrr et Craus (I977) ont d'ailleurs montré que la teneur en androsténone des graisses est reliée de façon plus étroite au poids relatif du tissu interstitiel.

\section{IV. - Relations avec les qualités organoleptiques des graisses et des viandes}

Dans le cas des 60 porcs mâles dont les graisses ont été jugées par le jury de laboratoire, le degré de liaison entre la teneur en androsténone des graisses et l'intensité des odeurs sexuelles $(r=+0,44 ; \mathrm{P}<0$, oor $)$ est du même ordre de grandeur que les valeurs établies par MaLmFors et ANDRESEN (I975), MaImFors, LundSTROM et HANSSON (I978) ou par NEWEr, et al. (I973). La faiblesse de ces relations provient d'abord de la variabilité résultant du caractère subjectif des notations d'odeur mais également de la contribution probable d'autres stéroïdes apparentés à l'androsténone (Thompson, PEARson et BANks, I972) ou du scatol (Vold I970, MaArse, Moerman et Walstra, I972).

L'acceptabilité des côtelettes des I7 porcs mâles entiers de la dernière répétition a été notée par un jury de type familial. Pour les teneurs en androsténone supérieures à I ppm, les réponses traduisent une augmentation importante des critiques concernant l'odeur de cuisson (57 p. Ioo d'avis défavorables) et à un moindre degré l'arôme ( 38 p.roo) et la saveur (46 p. roo) des viandes. 


\section{Conclusions}

L'abattage au stade commercial de roo $\mathrm{kg}$, d'animaux très jeunes ayant présenté une croissance très rapide ne permet pas d'éviter l'existence d'une proportion élevée d'individus dont les viandes présentent des risques importants de défauts d'odeur lors de la consommation en frais.

La teneur en androsténone du tissu adipeux ne permet certes pas d'expliquer l'ensemble des défauts d'odeur des viandes de pores mâles entiers. Son importance quant à la manifestation des odeurs sexuelles est cependant fondamentale. En vue d'expliquer la forte variabilité individuelle des teneurs mesurées, les recherches sur ce composé devront être menées selon deux axes d'orientation. D'une part les facteurs agissant sur le niveau de production d'androsténone doivent être mis en évidence, en liaison avec l'instauration de la maturité sexuelle. Par ailleurs, les relations entre la synthèse et le stockage de ce stéroïde restent à élucider.

Accepté pour publication en mars 1979.

\section{Remerciements}

Nous tenons à remercier MM. PAWLAk et BEUzEBOC de la Société I)uquesne Purina pour leur précieuse collaboration.

\section{Summary}

\section{Backfat androstenone content in entire male pigs of the "Camborough" line}

Backfat androstenone content was measured in 77 entire male pigs of the "Camborough" line, slaughtered at about 1 oo $\mathrm{kg}$ live weight and around $\mathrm{r}^{\circ}$ days of age. Androstenone level below I ppm, between I and 2 ppm and above 2 ppm concern 58,30 and I 2 p. I oo respectively of the animals (fig. I). There is no significant relationship between androstenone level on the one hand and liveweight, testes weight, body composition on the other hand. Because of the relationship between androstenone level and boar taint intensity, a large percentage of fresh pork may present matked odour defects, in spite of the young age of animals.

\section{Références bibliographiques}

ANDRESEN O., 1976. Concentration of fat and plasma $5 \alpha$ androstenone and plasma testosterone in boars selected for rate of body gain and thickness of backfat during growth, sexual maturation and after mating. J. Reprod. Fert., 48, 5I-59.

ANDRESEN O., BAKKE H., 1975. $5 \propto$ androstenone in fat from boars selected for rate of gain and thickness of backfat, and from boras used in artificial insemination service. Acta Vet. Scand., 16, 492-502.

Bonneau M., TAssfincourt I,, Desmoulin B., i975. Fitude de la réponse olfactive des juges en fonction des sites de chauffage des graisses de porcs mâles entiers, mâles castrés ou fennelles de race Large White. Journées Rech. Porcine en France, 7, 225-232, I.N.R.A., I.T.P. éd., Paris. 
Bonnfau M., Desmoti,in B., Pawiak M., 1977. Contrôle olfactif des odeurs sexuelles des viandes chez des jeunes porcs mâles entiers âgés de I 50 jours. Journées Rech. Porcine en France, 9, IoI-108, I.N.R.A., I.T.P. éd., Paris.

Bonneau M., Desmoulin B., Dumont B. I., I979. Qualités organoleptiques des viandes de porcs mâles entiers ou castrés : composition des graisses et odeurs sexuelles chez les races hypermusclés. Ann. Zootech., 28,53-72.

Burfsova M., Gutmann ri., I97I. E,ffect of testosterone on protein synthesis and contractility of the levator ani muscle of the rat. $J$. Endocr., 50, 643-65I.

CLAUS R., I974. Dosage radioimmunologique du $5 \propto$ androst-I6-ene-3-one, steroïde responsable de l'odeur de verrat, dans le tissu adipeux des porcs. C. R. Acad. Sc. Paris, 278, 299-302.

CI,AUS R., r 975. Messung des Ebergeruchstoffes im Fett von Schweinen mittels eines Radioimmunotests. r. Mitteilung: Geruchsdepotbildung in Abhängigkeit von Alter. Z. Tievzüchtg. Züchtungsbiol., 92, I $18-126$.

CLAUS R., I977. Phenomone bei Säugetieren unter besonderer Berücksichtigung des Ibergeruchsstoffes und Seiner Beziehung zu anderen Hodensterö̈den. Thèse de l'Université Technique de Munich, $329 \mathrm{p}$.

Desmovisn B., Rhodes D. N., I975. I, a production et l'utilisation des viandes de porc mâle entier. Bulletin Technique d'Information, 298, 265-276.

Dorst J., Sajonski H., I974. Zut Quantität der Gewebskonponenten des Schweinehodens während der postnatalen Entwicklung. Mh. Vet. Med., 17, 804-906.

IISFNBERG A., GORDON G. S., I950. The levator ani muscle of the rat as an index of myotrophic activity of steroidal hormones. J. Pharmacol. Fixp. Therap., 99, 38-44.

Grigsby J. S., Bercen W. G., Mikkki R. A., i976. The effect of testosterone skeletal muscle development and protein synthesis in rabbit. Cirowth, 40, 303-3 I6.

(iRoth W., Claus R., 1977. Beziehugen /wischen den Konzentrationen von Testosteron und dem Ebergeruchstoff $5 \alpha$ androst-I6-en-3-on in I3lut bzw. Fett gewebe und histometrischen Befunden im hoden vom Schwein. 7 bl. Vet. Med. A., 24, 103-12 I.

MAARSE H., MoERMAN P. C., WALSTRA P., I972. (Gaschromatografish en organoleptisch onderzoek van vless en spek van manvelijke varkens en van daarme bereide rookworst IVO Rapport CI 80 et rapport no 3 Research groep vlees en vleestearen TNO.

Malmfors B., AndResen O., I975. Relationship between boar tain intensity and concentration of $5 \propto$ androst-I 6ene-3-one in boar peripheral plasma and backfat. Act. A gri. Scand., 25, 92-96.

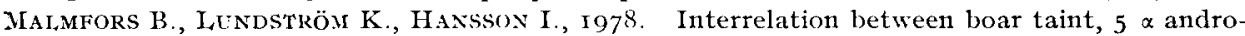
stenone and fatty acid composition in pigs. J. Agric. Res., 8, 16I-I69.

NFwei J. A., TuCkER L. H., STINSON (;. C., Bowland J. P., I973. Influence of late castration and diethylstilbestrol inplantation on performance of boars and on incidence of boar taint. Can. J. anim. Sci., 53, 205-210.

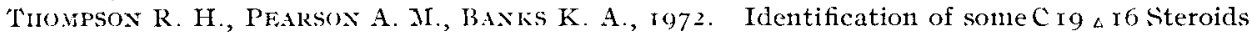
contributing to sex odor in pork. J. Agric. Fid. (hem., 20 (2), I95-I89.

VOL,D E., I97\%. Fleischproduktionseigenschaften bei Fbern und Kastraten IV Organoleptische und gaschromatografische Untersuchungen wasserdampfliichtiger Stoffe des Rückenspeckes von Ebern. Meld. Nowg. Landbrukshoegsk., 49, I-25.

WALKFR N, ROIBB D., PATTYKSON I). C., I 977 . The effect of grow th rate and sexual stimulation on the odour of boar fat. Anim. I'rod., 24, I 45 (Abst). 